\title{
Prenatal Methadone Exposure and Neonatal Neurobehavioral Functioning
}

\author{
MARTHA L. VELEZ, LAUREN M. JANSSON, JENNIFER SCHROEDER, AND ERICA WILLIAMS
}

\begin{abstract}
Department of Pediatrics [M.L.V., L.M.J., E.W.], Johns Hopkins University School of Medicine, Baltimore, Maryland 21224; Schroeder
\end{abstract} Statistical Consulting [J.S.], Ellicott City, Maryland 21042

\begin{abstract}
Opioid-exposed infants display a wide and variable range of dysregulated neurobehavioral functioning, but the regulatory difficulties experienced by these infants outside the defined clusters of neonatal abstinence syndrome (NAS) have not been well described and may have implications for the infant's developmental course. This study describes the neurobehavioral functioning of neonates prenatally exposed to methadone, using the NICU Network Neurobehavioral Scale (NNNS), and explores the relationships between maternal factors and infant functioning. The relationship between NNNS measures, NAS severity, and need for pharmacotherapy for NAS was also evaluated. Infants who required pharmacologic treatment for NAS showed more dysregulated behavior and signs of stress and abstinence as indicated by NNNS scores, but NNNS scores were not significantly correlated with maternal methadone dose. The determination of the range of the methadone-exposed infant's neurobehavioral repertoire could guide the optimal treatment of all such infants, particularly those requiring only nonpharmacologic care. (Pediatr Res 66: 704-709, 2009)
\end{abstract}

$\mathrm{O}$ pioid dependency remains a significant public health concern in the United States, and there is mounting evidence that problems related to abuse of opioid pain relievers and narcotic analgesics, such as hydrocodone and oxycodone, are increasing (1). Opioid dependency during pregnancy is a particular concern because there are significant consequences for the maternal-fetal/infant dyad. Methadone maintenance treatment remains the standard of care for opioid-dependent pregnant women in the United States. Methadone maintenance in the setting of comprehensive service provision during pregnancy significantly improves pregnancy outcomes for opioid-dependent women (2) but is not without consequences for the infant. Methadone crosses the placental barrier freely and acts diffusely in the fetal brain, potentially affecting several regions and processes that can result in variable and unpredictable behavioral deficits in the infant (3). The compromised nervous system in the methadone-exposed newborn negatively impacts the newborn's regulatory capacities (e.g. intensity of arousal, sensory, motor, autonomic regulation), challenging the adaptation of the newborn to extrauterine life and affecting basic functions, such as sleeping, feeding, thriving, and interactions with caregivers and the

Received March 5, 2009; accepted August 11, 2009.

Correspondence: Lauren M. Jansson, M.D., The Center for Addiction and Pregnancy, JHBMC, Department of Pediatrics, Johns Hopkins University School of Medicine, 4940 Eastern Avenue, D-5, Baltimore, MD 21224; e-mail: ljansson@jhmi.edu

Supported by NIH/NIDA Grant RO1DA019934 (to L.J.). environment (4,5). Evaluations of the difficulties presented by the opioid-exposed infant have been chiefly focused on the infant's display of neonatal abstinence syndrome (NAS), a cluster of signs and symptoms of dysfunction in several arenas, including central and autonomic nervous systems, gastrointestinal and respiratory systems, and the requirement for pharmacologic treatment of such syndromes (6). The regulatory difficulties experienced by these infants outside the defined clusters of symptoms of NAS have not been well described. Previous research, using the Brazelton Neonatal Behavioral Assessment Scale (NBAS; 7), on a group of methadone-exposed infants found them to be more state labile, displaying more tremors and hypertonicity than nonexposed infants; they cried more and had variable patterns of responses to stimulation (4). Behavioral management of symptoms of regulatory dysfunction displayed by the infant with NAS has been facilitated using the NBAS. In one study, a group of hyperirritable infants were treated successfully by applying the consolability domain techniques of the NBAS; the authors concluded that systematic observation of newborn behaviors could be a significant adjunct in diagnosis and management of NAS (4). On the basis of the NBAS, the NICU Network Neurobehavioral Scale (NNNS; 8) was developed as a neurobehavioral assessment for the at-risk infant. The NNNS has been used to evaluate the manners in which stressors, such as in utero substance exposure, maternal depression, and premature birth, affect infant self-organizing neurobehavioral capacities (9). There have been several studies that used the NNNS to describe the neurobehavioral effects of in utero exposure to cocaine $(10,11)$, nicotine (12), marijuana (13), and methamphetamine (14) exposure; however, assessment of opioidexposed newborns using the NNNS has been scarce $(15,16)$.

The recognition of regulatory difficulties expressed by opioid-exposed infants serves many purposes. Most important among them is the determination of the nature of the neurologic and behavioral dysfunction displayed, beyond the defined NAS symptom clusters. The determination of the range of infant's experience of dysregulated behavior could guide the optimal treatment of all such infants, particularly those requiring only nonpharmacologic care. In addition, the early detection of specific areas of dysregulation may indicate in-

\footnotetext{
Abbreviations: NAS, neonatal abstinence syndrome; NBAS, Neonatal Behavioral Assessment Scale; NNNS, NICU Network Neurobehavioral Scale; SSRI, selective serotonin reuptake inhibitor
} 
terventions that may ameliorate developmental, behavioral, or interactional problems.

The purpose of this study is to describe the neurobehavioral functioning of neonates prenatally exposed to methadone, using the NNNS on the infant's third day of life. The relationship between the NNNS and several maternal factors is explored, including maternal history of drug use, maternal methadone exposure, and other substance (illicit drug, nicotine, psychotropic medications) exposure. The relationship between NNNS measures, NAS severity, and need for pharmacotherapy for NAS is evaluated.

\section{METHODS}

Participants were 77 methadone-maintained pregnant women enrolled in a comprehensive treatment program offering mental health and substance abuse treatment, obstetric and pediatric care, described elsewhere (17), who delivered an infant free of significant medical complications, such as preterm $[<37$ wk estimated gestational age (EGA)] birth or need for medical interventions, and selected from an ongoing study evaluating the effects of methadone on maternal and fetal physiology, between 2002 and 2007. Maternal participants were enrolled at $32 \mathrm{wk}$ of gestation based on their ability to maintain drug abstinence and program compliance. All participants met DSM-IV-R (Diagnostic and Statistical Manual of Mental Disorders, 4th edition, revised) criteria for opiate dependence and federal guidelines regarding methadone maintenance therapy. Women with concurrent alcohol use as assessed by the Addiction Severity Index (18) interview on program intake, complications of pregnancy, such as gestational diabetes, multiple gestations, or significant health concerns (i.e. human immunodeficiency virus infection) that might affect newborn behavior were excluded from the study population. Program standards required, at a minimum, weekly random urinalysis testing for opiates, cocaine, benzodiazepines, barbiturates, THC, PCP, and alcohol. Other methodological details regarding the parent study have been described (19).

Maternal drug use, methadone history, and other substance exposure. Several measures of maternal substance exposure (licit substance abuse/illicit use and prescribed medications) were obtained to determine their association with the infant's neurobehavioral assessment on $d 3$, including maternal methadone history, licit/illicit drug use, psychotropic medications, and nicotine use. Maternal methadone maintenance history (trimester methadone maintenance initiated; total number of gravid days methadone received; total amount of methadone ingested during pregnancy; methadone dose at delivery) was obtained from medical records. Maternal drug use history was obtained from two sources: the total years of regular (3 times a week or more) drug use was gathered by subject report and percent of drug-positive urines from admission to delivery was obtained from medical records. Exposure to psychotropic medications, assessed by patient report at 36-wk EGA and confirmed by patient chart review, and nicotine (number of cigarettes smoked per day by patient report) was recorded for each participant. The Johns Hopkins Medicine Institutional Review Board 5 approved the protocol, and all subjects provided written, informed consent.

Infants. Infants were hospitalized for a minimum of $4 \mathrm{~d}$ postnatally for observation for signs/symptoms of NAS, as per standard care at the hospital of delivery. Infants received NAS scoring every 3-4 h for their entire hospitalization and were evaluated and treated based on an algorithm modified from Finnegan et al. (20) and described elsewhere (21). Treatment was begun with two consecutively obtained scores greater than 8 . Neonatal treatment at the hospital of record is a symptom-based algorithm that provides oral morphine based on severity of NAS symptoms. Increasing doses of medication are provided for escalating NAS scores until the infant achieves a plateau of scores less than 9. The infant is stabilized on this dose of medication for $48 \mathrm{~h}$ with all NAS scores less than 9 and then gradually weaned from medication. There are standardized protocols for weaning and re-escalation (i.e. if the infant requires more medication because of increasing NAS scores once stabilized). NAS scores were obtained by clinical nursing staff experienced in the treatment of drug-exposed neonates, who were blinded to the infant's enrollment in the study for the period of the infant's hospitalization. All infant hospitalization data were collected by chart review after discharge.

Neurobehavioral assessment. Infants $(n=77)$ underwent NNNS testing on the third day of life, chosen because most infants exhibiting significant symptoms of NAS do so by d 3 (6) and defined as the third block of $24 \mathrm{~h}$ after birth.
Developed for a multisite, longitudinal study of drug-exposed infants (22), the NNNS is a semi-structured, comprehensive instrument that assesses neonatal neurologic integrity and behavioral functioning, drug withdrawal, and general stress signs (8). The NNNS items are summarized into 13 separately scored subscales: habituation, attention, handling, quality of movement, regulation, nonoptimal reflexes, asymmetric reflexes, arousal, hypertonicity, hypotonicity, excitability, lethargy, and stress and abstinence. The stress and abstinence subscale includes a rating of the presence or absence of 50 signs of stress and abstinence grouped into seven categories: physiologic, autonomic, CNS, skin, visual, gastrointestinal, and state. The NNNS was administered in a quiet exam room, between feedings, with the infant initially asleep and swaddled. Examiners (M.L.V. and L.M.J.) were NNNS certified and blinded with respect to NAS treatment status.

Data analysis. Sociodemographic and clinical characteristics of the mothers and their infants were described using frequency distributions for categorical variables and mean/standard deviations for continuous variables. Because the NNNS measures were not normally distributed, nonparametric methods were used to evaluate the association between NNNS scores and 1) NAS scores; 2) the infant currently receiving pharmacologic treatment for NAS on d 3; and 3) maternal substance exposure variables, including methadone during pregnancy. A Spearman correlation matrix was used to evaluate the influence of maternal methadone use (i.e. methadone dose at delivery, gestational cumulative methadone dose, and number gravid days on methadone) on the NNNS scores. The influence of licit [nicotine, selective serotonin reuptake inhibitors (SSRI)] and illicit drug use on the NNNS was evaluated using a Spearman correlation matrix, with the goal of determining their possibility of being a confounding factor on the neurobehavioral effects of methadone use. Subsequently, partial Spearman correlations were used to reassess the degree of association between methadone use and NNNS variables controlling for other drug exposures (i.e. years of regular illicit drug use, cigarettes per day, and percent drug-positive urines). Finally, given that the need for pharmacologic treatment of NAS is an indicator of the severity of NAS and a possible surrogate indicator of the role of genetic and/or environmental factors that may alter the susceptibility to or expression of the methadone teratogenic process, we compared the NNNS scores of the infants who required pharmacologic treatment for NAS with those of infants who did not require pharmacologic treatment for NAS, using nonparametric one-way ANOVAs. Because of the non-normal distributions of NNNS variables, a Spearman correlation matrix was used to assess the degree of association between the NNNS summary scores and the NAS scores at $\mathrm{d} 3$.

\section{RESULTS}

Maternal characteristics are presented in Table 1. Mothers were predominantly white, in their late 20 s, and had less than a high school education. Most (86\%) participants listed heroin as their primary drug of choice. Most (79.3\%) participants began methadone treatment in the second trimester or earlier; mean methadone dose at delivery was $75.6 \pm 21.0 \mathrm{mg} / \mathrm{d}$. Twenty-one (27.3\%) participants were taking prescribed SSRI medications (i.e. fluoxetine, sertraline, and/or paroxetine) at the time of delivery for the treatment of psychiatric comorbidities, primarily depression.

Neonatal characteristics are presented in Table 2. A preponderance of female infants (65\%) was noted. Gestational age at delivery was defined by second trimester ultrasound, provided as a standard of care in the treatment program. Slightly more than half $(53.3 \%, n=41)$ of the participants required pharmacologic treatment for NAS. On average, the highest NAS score was 11 and was recorded on the third day of life. For neonates treated for NAS, the total number of days treated were $13.8 \pm 9.7$ (range 4-44).

A Spearman correlation matrix was generated to assess the degree of association between maternal methadone exposure and the NNNS scores. No significant correlations between methadone dose at delivery or gestational cumulative methadone dose and NNNS variables were observed. Days on 
Table 1. Maternal characteristics during pregnancy $(\mathrm{n}=77)$

\begin{tabular}{lc}
\hline & $n(\%) /$ Mean $(\mathrm{SD})$ \\
\hline Race & $46(60)$ \\
Caucasian & $28(36)$ \\
African American & $3(4)$ \\
Other/unknown & $27.9 \pm 5.5$ (range 18-41) \\
Age (y) & $11.0 \pm 1.5$ (range 6-14) \\
Education level (y, $n=75)$ & \\
Maternal substance use & $95.7 \%$ heroin, 5.2\% cocaine \\
1st Drug of choice* & $66.7 \%$ cocaine, $15.0 \%$ heroin, 5.2\% benzo \\
2nd Drug of choice* & $9.0 \pm 5.6$ (range 1-28) \\
Years of regular use & \\
$\quad$ (any drug) & $10.7 \pm 7.1$ (range 0-30) \\
Percent drug-positive urines & (admission to delivery) \\
Cigarettes per day & (range 0-48) \\
Trimester started methadone & $35.1 \% 1$ st, $44.2 \%$ 2nd, 5.2\% 3rd, 15.6\% \\
& throughout pregnancy \\
Days pregnant on methadone & $158.7 \pm 64.8$ (range 8-276) \\
Methadone dose at delivery & $75.6 \pm 21.0$ (range 15-120) \\
Cumulative methadone dose & $11050.16 \pm 5943.9$ (range 1330-28290) \\
Use of SSRI & 21 (27.3\%) \\
\hline
\end{tabular}

* Percentages in descending order; only drugs having frequencies of $5 \%$ or greater are listed.

Table 2. Neonatal characteristics of methadone exposed newborns $(\mathrm{n}=77)$

\begin{tabular}{|c|c|}
\hline & $n(\%) /$ Mean $(\mathrm{SD})$ \\
\hline \multicolumn{2}{|l|}{ Gender } \\
\hline Male & $27(35)$ \\
\hline Female & $50(65)$ \\
\hline Gestational age at delivery (wk) & $39.5 \pm 1.2($ range $36.6-41.4)$ \\
\hline Birth weight $(\mathrm{g})$ & $3116.4 \pm 501.9($ range $1885-4925)$ \\
\hline \multicolumn{2}{|l|}{ Neonatal withdrawal } \\
\hline Treated for opiate withdrawal & $41(53.3 \%)$ treated \\
\hline Highest Finnegan score & $11.1 \pm 4.7($ range $3-26)$ \\
\hline Day of highest Finnegan score & $3.2 \pm 2.3($ range $1-15)$ \\
\hline Average Finnegan score on d 1 & $3.7 \pm 2.2($ range $0.33-10.5)$ \\
\hline Average Finnegan score on d 2 & $6.0 \pm 2.8($ range $0.2-11.7)$ \\
\hline Average Finnegan score on d 3 & $5.9 \pm 2.4($ range $0.7-13.5)$ \\
\hline Length of hospital stay (d) & $10.5 \pm 9.0($ range $4-47)$ \\
\hline
\end{tabular}

methadone while pregnant were significantly inversely correlated with lethargy $(r=-0.31, p=0.0063)$.

Years of regular illicit drug use were significantly inversely correlated with the stress-skin category of the stress and abstinence NNNS subscale $(r=-0.27, p=0.02)$. Percent drug-positive urines from admission to delivery was significantly positively correlated with attention $(r=0.35, p=$ $0.0056)$ and negatively correlated with handling $(r=-0.24$, $p=0.0489)$ and stress state $(r=-0.31, p=0.0077)$. Nicotine exposure was not significantly associated with any NNNS variables.

Twenty-one mothers (27.3\%) were taking SSRIs. None of the differences in NNNS outcomes comparing mothers with and without SSRIs achieved statistical significance. SSRI use was not associated with NAS score (data not shown).

Partial correlations (Spearman) controlling for other exposures during pregnancy (cigarettes per day, percent drugpositive urines, and years of regular drug use) reveal that methadone dose at delivery and cumulative methadone dose were not significantly associated with any NNNS variables.
Number of gravid days on methadone was no longer associated with lethargy. However, gravid days on methadone became significantly positively correlated with self-regulation $(r=0.39, p=0.047)$ and quality of movement $(r=0.51, p=$ $0.0076)$ and inversely correlated with arousal $(r=-0.40, p=$ $0.042)$, excitability $(r=-0.46, p=0.019)$, and stress state $(r=-0.25, p=0.038)$.

NAS score was significantly correlated with arousal $(r=$ $0.23, p=0.041)$, self-regulation $(r=-0.31, p=0.0068)$, quality of movement $(r=-0.28, p=0.017)$, excitability $(r=$ $0.36, p=0.0013)$, hypertonicity $(r=0.49, p<0.0001)$, stress and abstinence $(r=0.32, p=0.0063)$, CNS stress $(r=0.29$, $p=0.013)$, and stress state $(r=0.28, p=0.017)$.

NNNS summary scores for the entire sample and for treated versus untreated newborns are shown below; comparison of scores was done using nonparametric one-way ANOVA. Infants requiring pharmacologic treatment had significantly higher scores for habituation $\left(F_{1,40}=4.14, p=0.048\right)$, arousal $\left(F_{1,75}=4.37, p=0.044\right)$, excitability $\left(F_{1,75}=5.70\right.$, $p=0.020)$, and hypertonicity $\left(F_{1,75}=6.05, p=0.016\right.$; Table 3 ) than infants not requiring pharmacologic treatment for NAS. Treated and untreated infants were not significantly different in terms of birth weight or gestational age; therefore, differences in these variables did not confound these analyses.

\section{DISCUSSION}

This study describes the behavioral repertoire and functioning of a group of newborns exposed to methadone in utero in relation to maternal history of substance abuse, exposure to methadone and/or other licit/illicit substances, and the infant's need for pharmacologic treatment for NAS.

In this sample, there were no significant correlations between the methadone dose at delivery or cumulative gestational methadone dose and the infants' neurobehavioral repertoire on d 3 of life as measured using the NNNS. These results support the findings from previous studies that suggested a lack of relationship between maternal methadone dose at delivery or cumulative gestational methadone and NAS severity as measured by the Finnegan scores (23-25), although this has been disputed by other researchers (26). However, results from the multivariate model in this study revealed that the number of gravid days on methadone might have a slightly positive effect on several areas of the newborn's functioning, such as self-regulation, quality of movement, arousal, excitability, and stress state on the NNNS. The reason for this finding may be related to the relationship between number of gravid days on methadone and length of time the mother spends in comprehensive drug treatment. In general, women engaged in treatment for longer periods of time are likely to have more prenatal and mental healthcare, less illicit drug use, and better lifestyles than women who receive less treatment, and these factors are likely to have a beneficial effect on the functioning of their infant.

As expected, this study confirms that infants who require pharmacologic treatment for NAS as measured by modified Finnegan scores have more dysregulated behavior and signs of stress and abstinence, as indicated by NNNS scores. Although 
Table 3. Neurobehavioral performance reflected by the summary scores of the NNNS for entire sample and NAS treated vs untreated infants

\begin{tabular}{lccc}
\hline & Entire sample $(N=77)$ & Treated newborns $(n=41)$ & Untreated newborns $(n=36)$ \\
\hline Habituation & $5.95 \pm 1.91(1.00-9.00)$ & $\mathbf{6 . 5 9} \pm \mathbf{1 . 3 9}(\mathbf{3 . 0 0}-\mathbf{8 . 3 3})$ & $\mathbf{5 . 4 3} \pm \mathbf{2 . 1 4}(\mathbf{1 . 0 0 - 9 . 0 0 )}$ \\
Attention & $3.09 \pm 1.61(1.00-7.71)$ & $2.96 \pm 1.49(1.00-7.14)$ & $3.26 \pm 1.76(1.20-7.71)$ \\
Arousal & $4.20 \pm 0.86(1.17-5.29)$ & $\mathbf{4 . 3 9} \pm \mathbf{0 . 8 1}(\mathbf{1 . 5 0 - 5 . 1 4})$ & $\mathbf{3 . 9 9} \pm \mathbf{0 . 8 8}(\mathbf{1 . 1 7 - 5 . 2 9 )}$ \\
Regulation & $4.83 \pm 0.77(3.23-6.47)$ & $4.77 \pm 0.75(3.36-6.20)$ & $4.89 \pm 0.80(3.23-6.47)$ \\
Handling & $0.80 \pm 0.23(0.25-1.00)$ & $0.82 \pm 0.21(0.38-1.00)$ & $0.76 \pm 0.26(0.25-1.00)$ \\
Quality of movement & $4.11 \pm 0.76(1.50-5.00)$ & $4.02 \pm 0.86(1.50-5.00)$ & $4.22 \pm 0.62(2.50-5.00)$ \\
Excitability & $4.22 \pm 2.76(0.00-11.0)$ & $\mathbf{4 . 9 0} \pm \mathbf{2 . 6 5}(\mathbf{0 . 0 0}-\mathbf{1 1 . 0})$ & $\mathbf{3 . 4 4} \pm \mathbf{2 . 7 0}(\mathbf{0 . 0 0 - 9 . 0 0})$ \\
Lethargy & $5.36 \pm 2.57(0.00-10.0)$ & $5.24 \pm 2.82(0.00-10.0)$ & $5.50 \pm 2.30(2.00-10.0)$ \\
Nonoptimal reflexes & $5.71 \pm 1.87(1.00-10.0)$ & $5.78 \pm 1.82(1.00-10.0)$ & $5.64 \pm 1.94(2.00-10.0)$ \\
Asymmetric reflexes & $1.36 \pm 1.53(0.00-7.00)$ & $1.66 \pm 1.81(0.00-7.00)$ & $1.03 \pm 1.06(0.00-4.00)$ \\
Hypertonicity & $0.74 \pm 1.12(0.00-6.00)$ & $\mathbf{1 . 0 2} \pm \mathbf{1 . 2 9}(\mathbf{0 . 0 0}-\mathbf{6 . 0 0})$ & $\mathbf{0 . 4 2} \pm \mathbf{0 . 8 0}(\mathbf{0 . 0 0 - 3 . 0 0})$ \\
Hypotonicity & $0.10 \pm 0.38(0.00-2.00)$ & $0.05 \pm 0.22(0.00-1.00)$ & $0.17 \pm 0.51(0.00-2.00)$ \\
Stress/abstinence & $0.22 \pm 0.08(0.04-0.45)$ & $0.23 \pm 0.08(0.08-0.39)$ & $0.21 \pm 0.08(0.04-0.45)$ \\
Stress-physiological & $0.16 \pm 0.31(0.00-1.00)$ & $0.18 \pm 0.35(0.00-1.00)$ & $0.14 \pm 0.26(0.00-1.00)$ \\
Stress-autonomic & $0.21 \pm 0.22(0.00-0.67)$ & $0.23 \pm 0.22(0.00-0.67)$ & $0.19 \pm 0.21(0.00-0.67)$ \\
Stress-CNS & $0.26 \pm 0.11(0.00-0.58)$ & $0.28 \pm 0.11(0.08-0.58)$ & $0.25 \pm 0.11(0.00-0.50)$ \\
Stress-skin & $0.17 \pm 0.16(0.00-0.67)$ & $0.17 \pm 0.18(0.00-0.67)$ & $0.17 \pm 0.15(0.00-0.67)$ \\
Stress-visual & $0.20 \pm 0.08(0.00-0.38)$ & $0.21 \pm 0.07(0.08-0.38)$ & $0.20 \pm 0.08(0.08-0.38)$ \\
Stress-GI & $0.16 \pm 0.21(0.00-0.67)$ & $0.16 \pm 0.21(0.00-0.67)$ & $0.17 \pm 0.22(0.00-0.67)$ \\
Stress state & $0.22 \pm 0.19(0.00-0.57)$ & $0.25 \pm 0.19(0.00-0.57)$ & $0.19 \pm 0.18(0.00-0.57)$ \\
\hline
\end{tabular}

Summary statistics for NNNS variables: mean \pm SD (range). Bold type indicates differences were statistically significant $(p<0.05)$.

infants not requiring treatment for NAS displayed more optimal performance in the majority of NNNS subscales, it is interesting to note that higher scores, particularly in the areas of arousal, excitability, and hypertonicity, were significantly different between the infants requiring versus those not requiring pharmacologic treatment. This suggests that infants requiring NAS treatment are more irritable, more hypertonic, and have greater difficulty modulating arousal. Difficulties in these areas may indicate the inability or difficulty of the infant in maintaining internal equilibrium while responding to external cues or stimulation. That the infant has difficulty in modulating sensory input from the environment, in addition to difficulty yielding appropriately organized responses, has important clinical implications both for interventions for the mother and infant in the neonatal period and for the early recognition of infant regulatory difficulties. Early identification and treatment of these difficulties may have a role in the prevention of subsequent long-term developmental and interactional problems. This is especially true for mothers already emotionally compromised by depression, anxiety, feelings of guilt, or insecurity that are potentially magnified by their knowledge that the infant will have a more severe course of NAS.

Although there were no significant associations between the maternal methadone dose at delivery and the NNNS subscale scores, there were significant neurobehavioral differences between infants requiring versus those not requiring pharmacologic treatment for NAS. These findings suggest that the variability in the severity of the symptoms displayed by the methadone-exposed neonate may be related to the interaction of methadone exposure with other factors, either maternal (e.g. prenatal stress) and/or fetal (e.g. genetic, nervous system maturational trajectory, gender, etc.). The identification of markers (clinical, neuroanatomic, neurophysiologic, or neurochemical) that are predictive of the subsequent expression of neonatal behavioral problems is crucial to the identification of particular prenatal and/or postnatal interventions that may prevent, offset, or modify the expression of functional maladaptations and their behavioral correlates (27). In addition, the association between the NNNS and NAS scores indicates that the NNNS is a tool capable of detecting those infants at risk for more severe NAS, and therefore earlier pharmacotherapeutic intervention. The combination of the tools in the evaluation of the exposed infant may allow earlier or more accurate identification of at-risk infants, and therefore earlier therapeutic intervention, possibly shortening the infant's hospital course.

Tronick et al. (28) described normative data for the NNNS by using a sample of selected 1- or 2-d-old healthy newborns who were not prenatally exposed to methadone or illicit substances. We compared the mean responses observed in our sample with their published data, using two-sample $t$ tests. The methadone-exposed newborns in this sample, even those not requiring pharmacotherapy for NAS, had different functioning in several NNNS subscales than the healthy nonexposed newborns in the domains of habituation, attention, handling, nonoptimal reflexes, hypertonicity, hypotonicity, and stress and abstinence, suggesting impairment in regulatory functioning among the methadone-exposed infants. This indicates that all opioid-exposed infants, even those not requiring pharmacotherapy for significant expression of symptoms, have specific difficulties in some neurobehavioral areas leading to specific care requirements in at least the neonatal period, which must be relayed to caregivers. Recognition of these problems may have a positive impact on the newborn's functioning, the mother-infant interaction, and complement the infant and maternal recovery process (29).

Use of illicit drugs in addition to methadone seems to play a role in the neurobehaviors displayed by the methadoneexposed newborn. In this sample, a higher percentage of any maternal illicit drug-positive urines during pregnancy was 
positively correlated with attention and inversely correlated with handling and stress state categories, suggesting that some of the variability in the neurobehavioral performance among this group may be related to the effects of other substances. The differential expression of neurobehaviors in infants exposed to both methadone and other drugs during gestation has been limited. A study using the NBAS comparing groups of methadone-exposed, polydrug (nonmethadone)-exposed and control (nonexposed) newborns found that the "polydrug" nonmethadone-exposed groups displayed neurobehaviors that were more dysregulated than controls but displayed less impairment in functioning than the methadone-exposed group. This suggests a possible escalating continuum of somatic and physiologic impairments, which range from normal nonexposed control newborns, to nonopioid-exposed infants of polydrug users to infants of methadone-maintained women (30). Scales, such as the NNNS, that measure aspects of infant neurobehavioral functioning differently from the Finnegan scales, may be more accurate in detecting the spectrum of difficulties presented by the methadone/polydrug-exposed newborn; in addition, methadone exposure should be considered as an indicator of exposure to multiple stressors (e.g. poor nutrition, late prenatal care, psychosocial problems, etc.) that can impact the newborn's behavior (10). Further studies are needed to better understand the interactive effects of these factors on the severity of symptoms and to address the surrounding context in which methadone maintenance occur during pregnancy.

Maternal SSRI use was not associated with NAS scores, which is consistent with previous work by this team of investigators (24). Interestingly, there were no associations between NNNS scores and cigarette use. Nicotine exposure has been found to potentiate NAS severity among infants of methadone-maintained heavy smokers (31), and nicotine alone has been shown to produce a withdrawal-like phenomenon in exposed infants (32). Further studies are needed to determine the nature of these effects, both in tandem with methadone exposure and individually.

Both human and animal studies indicate that prenatal methadone exposure produces a variety of short- and long-term neurobehavioral sequelae (33-35). Although the immediate postnatal impact of maternal methadone use on infants has long been recognized as NAS, there are still many unanswered questions related to the variability of functioning for this group of infants. Researchers and clinicians usually attribute the functional status of methadone-exposed newborns to NAS, as defined by the need for medication therapy only. However, scales that only explore NAS symptoms do not capture some aspects of the newborn's neurobehavioral repertoire that can impair the infant's regulatory functioning and interactions with the environment and caregivers. It is not completely understood whether these newborn behaviors are only direct effects of the abrupt withdrawal of the maternal opioid on delivery, but it seems from this research that other undescribed factors play a significant role in neonatal neurobehavioral expression.

There are several limitations in this study. First, these data reflect infant functioning using the NNNS on d 3 of life, and the bearing of these findings on the future development of affected infants is not known. Second, there are methadoneexposed infants who develop significant symptoms of NAS after d 3 of life. It is possible that some of the infants who were included in the nonpharmacologic subsample developed symptoms of NAS severe enough to require pharmacologic treatment after they were released from the hospital and were not included in the treated subsample. Third, the maternal use of other psychoactive substances (illicit drugs and SSRIs) in addition to methadone that may affect neonatal neurobehavior among the subjects in this sample. The population of infants studied was selected based on their mother's ability to maintain drug abstinence and program compliance, a sample that may not be highly generalizable to the general population of opioid-dependent, pregnant women. Similarly, the infants studied were born at term and free from significant medical complications other than NAS, which may also limit generalization to the population of methadone- and other drugexposed infants as a whole. Ultimately, any study describing infants of drug-dependent women will entail the confounding effects of early illicit drug exposure, licit drug exposure, and other factors common to drug-using populations.

This study suggests the need for a systematic evaluation of the broad range of the methadone-exposed infant's neurobehavioral repertoire using tools such as the NNNS, in whole or in part, in combination with the Finnegan scale. This comprehensive evaluation would include items not adequately captured by scales that evaluate NAS and that could impact the developmental, behavioral, and interactional trajectories of the infant. Information gleaned from a combination of tools may provide a better understanding of and, therefore, more optimal treatment for opioid-exposed infants, particularly those who have more subtle impairments in functioning that do not ultimately require pharmacologic treatment for NAS. Information from the NNNS can be used as part of the discharge plan and education of the caregivers (9) and, as such, may be highly beneficial to the recovery process of the chemically dependent woman.

Gaps in knowledge about the relationship between alterations in neurobehavioral functioning in young infants remain, such as those described in this study, and later functioning, particularly because longitudinal studies involving infants of drug-dependent women are difficult as a result of the multiple confounders often present in this group. It is not known whether the newborn's regulatory deficits portend a more persistent dysregulation that creates vulnerability for later developmental and behavioral problems. There is a need for longitudinal studies defining the developmental trajectories of methadone-exposed infants who display dysregulation in the neonatal period, to include the ability of the infant to overcome the early deficits in neurobehavioral functioning as well as the evaluation of interventions that can assist this population. By determining the specific nature of neurobehavioral functioning of infants exposed to methadone during pregnancy, we can optimize their pharmacologic and nonpharmacologic care and inform future investigations into long-term functioning of opioid-exposed infants. 


\section{REFERENCES}

1. Substance Abuse and Mental Health Services Administration (SAMHSA) Office of Applied Studies (OAS) 2008 Results from the 2007 National Survey on Drug Use and Health: National Findings. Rep No NSDUH Series H-34, DHHS Publication No SMA 08-4343. SAMHSA OAS, Rockville, MD

2. Gottheil E, Sterling RC, Weinstein SP 1993 Diminished illicit drug use as a consequence of long-term methadone maintenance. J Addict Dis 12:45-57

3. Yanai J, Huleihel R, Izrael M, Metsuyanim S, Shahak H, Vatury O, Yaniv SP 2003 Functional changes after prenatal opiate exposure related to opiate receptors' regulated alterations in cholinergic innervation. Int J Neuropsychopharmacol 6:253265

4. Soule AB, Standley K, Copans SA, Davis M 1974 Clinical uses of the Brazelton Neonatal Scale. Pediatrics 54:583-586

5. Velez M, Jansson LM 2008 The opioid dependent mother and newborn dyad: nonpharmacologic care. J Addict Med 2:113-120

6. 1998 Neonatal drug withdrawal. American Academy of Pediatrics Committee on Drugs. Pediatrics 101:1079-1088

7. Brazelton TB 1973 Neonatal Behavioral Assessment Scale: Clinics in Developmental Medicine. Spastics International Medical Publications, London

8. Lester BM, Tronick EZ 2004 The Neonatal Intensive Care Unit Network Neurobehavioral Scale procedures. Pediatrics 113:641-667

9. Tronick E 2007 The Neurobehavioral and Social-Emotional Development of Infants and Children, 1st ed. W.W. Norton \& Company, New York

10. Lester BM, Tronick EZ, LaGasse L, Seifer R, Bauer CR, Shankaran S, Bada H, Wright LL, Smeriglio VL, Lu J, Finnegan LP, Maza PL 2002 The maternal lifestyle study: effects of substance exposure during pregnancy on neurodevelopmental outcome in 1-month-old infants. Pediatrics 110:1182-1192

11. Napiorkowski B, Lester BM, Freier MC, Brunner S, Dietz L, Nadra A, Oh W 1996 Effects of in utero substance exposure on infant neurobehavior. Pediatrics 98:71-75

12. Law KL, Stroud LR, LaGasse LL, Niaura R, Liu J, Lester BM 2003 Smoking during pregnancy and newborn neurobehavior. Pediatrics 111:1318-1323

13. Moraes Barros MC, Guinsburg R, de Araujo PC, Mitsuhiro S, Chalem E, Laranjeira RR 2006 Exposure to marijuana during pregnancy alters neurobehavior in the early neonatal period. J Pediatr 149:781-787

14. Smith LM, LaGasse LL, Derauf C, Grant P, Shah R, Arria A, Huestis M, Haning W, Strauss A, Grotta SD, Fallone M, Liu J, Lester BM 2008 Prenatal methamphetamine use and neonatal neurobehavioral outcome. Neurotoxicol Teratol 30:20-28

15. Coyle MG, Ferguson A, LaGasse L, Liu J, Lester B 2005 Neurobehavioral effects of treatment for opiate withdrawal. Arch Dis Child Fetal Neonatal Ed 90:F73-F74

16. Jansson LM, Choo R, Velez ML, Harrow C, Schroeder JR, Shakleya DM, Huestis MA 2008 Methadone maintenance and breastfeeding in the neonatal period. Pediatrics 121:106-114
17. Jansson LM, Svikis D, Lee J, Paluzzi P, Rutigliano P, Hackerman F 1996 Pregnancy and addiction: a comprehensive care model. J Subst Abuse Treat 13:321-329

18. McLellan AT, Kushner H, Metzger D, Peters R, Smith I, Grissom G, Pettinati H, Argeriou M 1992 The Fifth Edition of the Addiction Severity Index. J Subst Abuse Treat 9:199-213

19. Jansson LM, Dipietro J, Elko A 2005 Fetal response to maternal methadone administration. Am J Obstet Gynecol 193:611-617

20. Finnegan LP, Connaughton JF Jr, Kron RE, Emich JP 1975 Neonatal abstinence syndrome: assessment and management. Addict Dis 2:141-158

21. Jansson LM, Velez M, Harrow C 2009 The opioid exposed infant: assessment and pharmacologic management. J Opioid Manag 5:47-58

22. Lester BM 1998 The Maternal Lifestyles Study. Ann N Y Acad Sci 846:296-305

23. Berghella V, Lim PJ, Hill MK, Cherpes J, Chennat J, Kaltenbach K 2003 Maternal methadone dose and neonatal withdrawal. Am J Obstet Gynecol 189:312-317

24. Jansson LM, DiPietro JA, Elko A, Velez M 2007 Maternal vagal tone change in response to methadone is associated with neonatal abstinence syndrome severity in exposed neonates. J Matern Fetal Neonatal Med 20:677-685

25. McCarthy JJ, Leamon MH, Parr MS, Anania B 2005 High-dose methadone maintenance in pregnancy: maternal and neonatal outcomes. Am J Obstet Gynecol 193:606-610

26. Dryden C, Young D, Hepburn M, Mactier H 2009 Maternal methadone use in pregnancy: factors associated with the development of neonatal abstinence syndrome and implications for healthcare resources. BJOG 116:665-671

27. Trask CL, Kosofsky BE 2000 Developmental considerations of neurotoxic exposures. Neurol Clin 18:541-562

28. Tronick EZ, Olson K, Rosenberg R, Bohne L, Lu J, Lester BM 2004 Normative neurobehavioral performance of healthy infants on the Neonatal Intensive Care Unit Network Neurobehavioral Scale. Pediatrics 113:676-678

29. Boukydis CF, Lester BM 2008 Mother-infant consultation during drug treatment: research and innovative clinical practice. Harm Reduct J 5:6

30. Chasnoff IJ, Hatcher R, Burns WJ 1982 Polydrug- and methadone-addicted newborns: a continuum of impairment? Pediatrics 70:210-213

31. Choo RE, Huestis MA, Schroeder JR, Shin AS, Jones HE 2004 Neonatal abstinence syndrome in methadone exposed infants is altered by level of prenatal tobacco exposure. Drug Alcohol Depend 75:253-260

32. Garcia-Algar O, Tuig C, Mendez C, Vall O, Pacifici R, Pichini S 2001 Neonatal nicotine withdrawal syndrome. J Epidemiol Community Health 55:687-688

33. de Cubas MM, Field T 1993 Children of methadone-dependent women: developmental outcomes. Am J Orthopsychiatry 63:266-276

34. Johnson HL, Rosen TS 1982 Prenatal methadone exposure: effects on behavior in early infancy. Pediatr Pharmacol (New York) 2:113-120

35. Robinson SE, Maher JR, Wallace MJ, Kunko PM 1997 Perinatal methadone exposure affects dopamine, norepinephrine, and serotonin in the weanling rat Neurotoxicol Teratol 19:295-303 